\title{
Attention-deficit hyperactivity disorder
} and life-span development

\author{
PAUL MCARDLE
}

Developmentally inappropriate degrees of hyperactivity, inattention and impulsivity are common symptoms in childhood. If of early onset (before 7 years), present in different settings and to a degree that causes functional impairment, a diagnosis of attention-deficit hyperactivity disorder (ADHD) or 'hyperkinetic disorder' should be considered. The age criterion is crucial to distinguish ADHD and hyperkinetic disorder from later-onset conditions such as agitated depression, adolescent-onset socialised conduct disorder (probably not linked to ADHD) or hypomania, that might appear superficially similar. Attention-deficit hyperactivity disorder and hyperkinetic disorder are commonly associated with language, learning and social impairments. Indeed, in severe cases hyperactivity could be a marker of a multifaceted developmental disorder. Hence, competent assessment usually requires a thorough history, often using a number of sources.

Attention-deficit hyperactivity disorder appears to represent a 'dysmaturity' of the prefrontal cerebral cortex and subcortical structures such as the basal ganglia. In addition, approximate phenocopies might also emerge from intra-uterine alcohol exposure, brain injury and, possibly, severe preschool social deprivation. However, it is likely that genetic factors contribute significantly to most cases of ADHD and hyperkinetic disorder in representative child populations.

\section{IMPORTANCE OF ADHD}

Such a biologically oriented view amounts to a major reconceptualisation of childhood behavioural disorder and has made a substantial impact on child and adolescent psychiatry. It could be on the brink of doing so for aspects of adult practice, not least because adult services are likely to be already treating individuals who have or have had
ADHD (Dalsgaard et al, 2002). Insights from child and adolescent practice are therefore likely to be relevant to general psychiatrists.

Nevertheless, the idea that ADHD is responsible for a large proportion of seriously disruptive childhood behaviour was accepted only slowly by UK clinicians, who had favoured explanations that were more socially oriented. Hence, conduct disorder, characterised by 'a repetitive... pattern of dissocial aggressive or defiant conduct' and regarded as subsequent to various forms of family adversity but without a significant biological predisposition, had tended to prevail.

However, aspects of both conduct disorder and ADHD could characterise the behaviour of the most disturbed children with the poorest prognosis. For this and other reasons ICD-10 included a new category, hyperkinetic conduct disorder (World Health Organization, 1992). It now appears likely that this is not simply two common disorders that occur together but a more severe variant of behavioural disorder with a greater genetic loading than ADHD alone.

\section{A CULTURE-BOUND SYNDROME?}

The prevalence of ADHD combined type (i.e. with both inattention and hyperactivity) or hyperkinetic disorder is approximately $1-2 \%$ of the primary school population. However, cultural factors appear to influence its prevalence (Leung et al, 1996) and severity (Lynam et al, 2000), a phenomenon familiar to general psychiatrists (Harrison et al, 2001). Indeed, some argue that in recent decades Western culture has tended to evolve to the disadvantage of children as well as other vulnerable groups (Murray et al, 2003). These include, for instance, the loss of extended and even nuclear families, informal social controls, safe play areas and, because of crime or traffic, the general freedom of the outdoors. For older children there is the added risk posed by ubiquitous access to affordable illicit drugs and alcohol.

Therefore, it could be that whereas the prevalence of ADHD as a genetically determined syndrome has not changed, there has been a decline in the capacity of Western culture to cope with and raise these children. The implication for the individual is greater culture-related handicaps and for society, greater comorbid or secondary conduct disorders (Jacobson et al, 2000) with all the social disruption, substance misuse and mortality (through substance misuse, accidents and suicide) that that entails.

An analogous set of secular changes might have also contributed to the increased salience of adult ADHD. Driving presents particular risks for young adults with ADHD. In addition, Western economies require higher levels of education, social competence and self-organisation than in even the fairly recent past (European Commission, 2000), the precise areas that ADHD impairs. Hence, although not culture bound, the significance of ADHD is likely to vary with context.

\section{COMORBIDITYAND LINKS WITH ADULT DISORDER}

In keeping with a developmental disorder, the prevalence of ADHD declines with age. Nevertheless, impulsivity, disorganisation, impaired concentration and poor planning can remain as disruptive symptoms. Although ADHD bears a superficial resemblance to hypomanic behaviour, follow-up studies do not seem to point to an increased rate of bipolar disorders (Mannuzza et al, 1998). Also, despite observations that children who are premorbid for schizophrenia bear neurodevelopmental vulnerabilities similar to ADHD, follow-up does not appear to show unusually high rates of schizophrenia (Mannuzza et al, 1998), nor are there raised rates of psychosis in the first degree relatives of children who are hyperactive. Nevertheless, in keeping with the functional impairments linked with the condition, there is considerable affective comorbidity.

The presence of comorbid conduct disorder, possibly because it marks a more severe condition, points towards the likelihood of persistence; approximately $20 \%$ of children referred to clinics with ADHD 
enter adulthood with antisocial personality disorder (Mannuzza et al, 1998). Interestingly, brain imaging studies suggest that, as with ADHD, individuals with antisocial personality disorder have frontal cortical deficits (Raine et al, 2000). Hence, there are grounds to believe that antisocial personality disorder could be an adult manifestation of unresolved hyperkinetic disorder.

\section{INTERVENTION}

Psychosocial therapies might be more effective for the educational, social and other comorbidities than for the core syndrome itself. Engagement with the child and family, or adult patient, the provision of accurate information, sustained support and advocacy, parenting or family interventions and educational measures are likely to be important. Although long-term follow-up studies of intervention are few, combined psychosocial interventions and medication probably additively enhance outcome and reduce risk for adolescent delinquency and substance misuse (Satterfield et al, 1987).

Among adolescents and adults presenting for the first time, evidence of early onset (a typical history, supported by old school reports) and a collateral history are generally helpful in discriminating true ADHD. Where choice exists, symptomatic adults might also benefit from the advice to seek out occupations that do not require sustained attention or indeed that can hold the patient's interest. Also, it is possible to indicate that because the frontal cortex continues to mature well into adult life (Bartzokis et al, 2001), maturation and improvement are likely to continue. Indeed, with such a familial condition it is often possible to point to other 'late developers' in the family. Explanations to patients and to their partners could contribute to mutual understanding, and preliminary data suggest that group or cognitive psychotherapy might have a useful role.

There is now solid evidence at least for the short-term efficacy of stimulant medication and for probably weaker, but still helpful, effects of tricyclic and other antidepressants for adult ADHD. Among

PAUL McARDLE, MB, FRCPI, FRCPsych, DCH, Fleming Nuffield Unit, Newcastle North Tyneside and Northumberland Mental Health Trust, Tyne House, Tankerville Terrace, Newcastle upon Tyne NE2 3BB, UK

(First received 14 May 2003, final revision 3 November 2003, accepted 5 November 2003)

young adults, stimulant treatment of ADHD comorbid with substance misuse benefits symptoms of ADHD (e.g. Upadhyaya et al, 2001). The possibility is that where ADHD symptoms are present, even among those with apparently intractable personality problems and even when comorbid with substance misuse, there could be an unrealised potential for treatment.

\section{CONCLUSIONS}

It is conceivable that ADHD conveyed valuable qualities in different times and, under certain circumstances, perhaps partial syndromes still do. However, in the contemporary Western context, or indeed among communities affected by the demands of globalisation, ADHD is attended by significant risks to well-being and health. It may symbolise a wider phenomenon; with changing culture, patterns or concepts of disease might change too. Doctors and other health care workers need to adapt to this or risk, conceivably, even losing the confidence of the populations they serve. Effective treatment, but also insight into the complex nature of $\mathrm{ADHD}$, and debate focused on its implications for the way we live, would be a usefully civilising medical and social advance.

\section{DECLARATION OF INTEREST}

P.M. has been invited to speak at pharmaceutical industry sponsored conferences, was sponsored to attend the American Academy of Child and Adolescent Psychiatry's annual meeting and is involved in a pharmaceutical industry randomised controlled trial.

\section{REFERENCES}

Bartzokis, G., Beckson, M., Lu, P., et al (200I) Agerelated changes in frontal and temporal lobe volumes in men: a magnetic resonance imaging study. Archives of General Psychiatry, 58, 46I-465.

Dalsgaard, S., Mortensen, P. B., Frydenberg, M. et al (2002) Conduct problems, gender and adult psychiatric outcome of children with attention-deficit hyperactivity disorder. British Journal of Psychiatry, $\mathbf{1 8 1}$, 416-421.

European Commission (2000) Building an Inclusive Europe. http: / /europa.eu. int/eur-lex/en/com/cnc/ 2000/com2000_0079en02.pdf

Harrison, G., Hopper, K., Craig, T., et al (200I) Recovery from psychotic illness: a 15- and 25-year international follow-up study. British Journal of Psychiatry I78, 506-517.

Jacobson, K., Prescitt, C., Neale, M., et al (2000) Cohort differences in genetic and environmental influences on retrospective reports of conduct disorder among adult male twins. Psychological Medicine, 30, 775-787.

Leung, P. W., Luk, S. L., Ho, T. P., et al (1996) The diagnosis and prevalence of hyperactivity in Chinese schoolboys. British Journal of Psychiatry, 168, 486-496.

Lynam, D., Caspi, A., Moffitt, T., et al (2000) The interaction between impulsivity and neighborhood context on offending: the effects of impulsivity are stronger in poorer neighborhoods. Journal of Abnormal Psychology, 109, 563-574.

Mannuzza, S., Klein, R., Bessler, A., et al (1998) Adult psychiatric status of hyperactive boys grown up. American Journal of Psychiatry, I55, 493-498.

Murray, S., Grant, E., Grant, A., et al (2003) Dying from cancer in developed and developing countries: lessons from two qualitative interview studies of patients and their carers. BMJ, 326, 345-404.

Raine, A., Lencz, T., Bihrle, S., et al (2000) Reduced prefrontal gray matter volume and reduced autonomic activity in antisocial personality disorder. Archives of General Psychiatry, 57, 119-127.

Satterfield, J. H., Satterfield, B. T. \& Schell, A. (1987) Therapeutic interventions to prevent delinquency in hyperactive boys. Journal of the American Academy of Child and Adolescent Psychiatry, 26, 56-64.

Upadhyaya, H., Brady, K., Sethuraman, G., et al (200I) Venlafaxine treatment of patients with comorbid alcohol/cocaine abuse and attention deficit/ hyperactivity disorder: a pilot study. Journal of Clinical Psychopharmacology, 21, 116-118.

World Health Organization (1992) ICD-10 Classification of Mental and Behavioural Disorders. Geneva: WHO. 